\title{
APRIL Induces Cisplatin Resistance in Gastric Cancer Cells via Activation of the NF-kB Pathway
}

\author{
Xiaofei Zhi ${ }^{a}$ Jinqiu Tao ${ }^{d}$ Guoliang Xiang ${ }^{b}$ Hongyong Cao ${ }^{c}$ Zijun Liuc \\ Kunxing Yang ${ }^{c}$ Chengyu $\mathrm{Lv}^{c}$ Shaozhong $\mathrm{Ni}^{c}$ \\ aDepartment of General Surgery, the First Affiliated Hospital of Nanjing Medical University, Nanjing, \\ ${ }^{b}$ Department of General Surgery, Xunyang Chinese Medicine Hospital, Ankang, 'Department of General \\ Surgery, Nanjing First Hospital, Nanjing Medical University, Nanjing, 'Department of General Surgery, \\ Nanjing Drum Tower Hospital, the Affiliated Hospital of Nanjing University Medical School, Nanjing, \\ China
}

\author{
Key Words \\ APRIL • Gastric cancer • Cisplatin resistance $・$ NF-kB pathway
}

\begin{abstract}
Background: A proliferation-inducing ligand (APRIL) is a tumor-necrosis factor (TNF) family member and is a novel cytokine crucial in sustaining lymphocytic leukemia B cell survival and proliferation. However, its role in gastric cancer (GC) remains unclear. In this study, we investigated the expression pattern and prognostic role of APRIL in GC. Methods: Expression of APRIL was assessed by immunohistochemistry and real-time PCR. Prognostic role of APRIL expression was evaluated. We also discovered the effect of APRIL on chemo-resistance in GC cells and the underlying mechanisms. Results: APRIL mRNA levels were significantly increased in GC tissues compared with adjacent tissues and high expression levels of APRIL in tumor cells significantly correlated with poor overall survival in patients receiving cisplatin adjuvant treatment. Overexpression of APRIL in AGS cells significantly attenuated the therapeutic efficacy of cisplatin in vitro and in vivo. In contrast, silence of APRIL in SGC7901 cells enhanced cisplatin-induced tumor suppression. Our data further revealed that the canonical NF-KB pathway was involved in APRIL-mediated chemo-resistance. In addition, expression of APRIL was regulated by miR-145 in GC cells. Conclusion: APRIL is a novel clinical chemo-resistance biomarker for gastric cancer and might be a promising therapeutic target for GC patients.
\end{abstract}


Zhi et al.: APRIL Expression in Gastric Cancer

\section{Introduction}

Gastric cancer (GC) is the fourth leading cancer in 2008 worldwide [1]. Despite the improvement of adjuvant chemotherapy after surgery, the disease is still the second most frequent cause of cancer-related death. Identification of biological markers that determine the sensitivity of gastric cancer cells to chemotherapy is necessary to improve the prognosis. Cisplatin (CDDP) is a first-line chemotherapeutic agent in the perioperative treatment of GC [2]. However, the underlying mechanisms of cisplatin resistance in GC remain largely unknown.

A proliferation-inducing ligand (APRIL, also known as TNFSF13, CD256) is a tumornecrosis factor (TNF) family member, expressed by a variety of cells containing neutrophils, monocytes, macrophages, but also epithelial cells and cancer cells [3-5]. APRIL acts as a novel cytokine crucial in sustaining B cell survival and proliferation by binding to its BCMA (TNFSF13C, CD269) and TACI (TNFSF13B, CD267) receptors [6, 7]. As recently reported, not only APRIL is expressed in a wide array of B cell malignancies, but high levels of APRIL expression in tissue of these patients correlate with poor prognosis [8]. Subsequent studies demonstrated that APRIL was able to protect B cell malignant cells from drug-induced apoptosis $[9,10]$. High levels of APRIL mRNA are also detectable in human colorectal, hepatocellular, ovarian, bladder and head and neck carcinomas [11-14]. Furthermore, cancers of gastrointestinal tract express particularly high levels of APRIL mRNA [3]. These findings suggest a broad and important function of APRIL ranging from B cell malignancies to solid tumors, especially in gastrointestinal cancers. However, there are few reports regarding the role of APRIL in gastric cancer. Our previous study revealed that knockdown of APRIL promoted apoptosis of gastric cancer cells and down-regulated the receptor tyrosine kinases [15]. In the present study, we investigated the mRNA and protein expression levels of APRIL in gastric cancer and paired-adjacent gastric mucosa. Our data demonstrated that APRIL was significantly up-regulated in gastric cancer and high levels of APRIL correlated with resistance to cisplatin. The underlying mechanism was the activation of the canonical $\mathrm{NF}-\kappa \mathrm{B}$ pathway. These results indicated that the gastric cancer patients might benefit from the APRIL-targeting agents combined with chemotherapy.

\section{Materials and Methods}

\section{Ethics statement}

The study protocols were approved by the Ethical Committee of the First Affiliated Hospital of Nanjing Medical University. All animal work was approved by the Ethical Committee of the First Affiliated Hospital of Nanjing Medical University. Written informed consents were obtained before specimen collection.

\section{Patients and specimens}

Paired tumorous and adjacent non-tumorous human gastric tissues were obtained from 159 patients with gastric cancerwho underwent radical resection in Affiliated Nanjing Hospital, Nanjing Medical University, from 2006 to 2007. All patients were diagnosed pathologically according to the criteria of the American Joint Committee on Cancer by two professional pathologists independently. Among the 159 patients, 148 received 5-FU adjuvant chemotherapy while 129 received cisplatin regimen. Clinicopathological details are provided in Table 1. Overall survival (OS) was the primary end-point. Survival time was calculated from the date of surgery to the date of death or the last follow-up.

Immunohistochemistry and assessment

Immunohistochemistry (IHC) was carried out as described previously [16]. A polyclonal rabbit anti-APRIL (Abcam) was used. Staining of APRIL in tumorous and non-tumorous tissue was scored independently by two pathologists using a semi-quantitative immunoreactivity score (IRS) [17]. The IRS was calculated by combining the quantity score with the intensity score. The quantity score documented the percentage of immunoreactive cells as 1 (0-25\%), 2 (26-50\%), 3 (51-75\%), and 4 (76-100\%). The intensity score documented the intensity of immunostaining as 0 (negative), 1 (weak), 2 (moderate) and 
Zhi et al.: APRIL Expression in Gastric Cancer

Table 1. Clinicopathological details of 159 gastric cancer patients. athe $7^{\text {th }}$ AJCC staging manual

\begin{tabular}{lcc}
\hline characteristics & No. of patients & $\%$ \\
\hline Total patients & 159 & 100 \\
Gender (male/female) & $98 / 61$ & $61.6 / 38.4$ \\
Age, year (median, range) & $51,31-78$ & \\
Tumor size ( $\geq 3 \mathrm{~cm} /<3 \mathrm{~cm})$ & $84 / 75$ & $52.8 / 47.2$ \\
Differentiation (moderately and poorly/ well) & $103 / 56$ & $64.8 / 35.2$ \\
${\text { TNM stage }(\mathrm{I}+\mathrm{II} / \mathrm{III}+\mathrm{IV})^{\mathrm{a}}}^{\mathrm{a}}$ & $18 / 141$ & $11.3 / 88.7$ \\
\hline
\end{tabular}

3 (strong). Multiplication of quantity score and intensity score resulted in an IRS ranging from 0 to 12. The optimal cutoff value of IRS was calculated by receiver-operator characteristic (ROC) analysis [18]. The values of area under curve (AUC) at different IRS of APRIL for OS were obtained. The optimal cutoff value of IRS was 3 (Fig. 2D). So the samples with IRS 0-3 were classified as low APRIL expression and the samples with IRS 4-12 were classified as high APRIL expression, respectively.

\section{Quantitative real-time PCR}

Total RNA was extracted with the TRIzol reagent (Invitrogen) according to the manufacturer's instructions, and was reverse transcribed into cDNA using Primescript RT Reagent (Takara). Realtime PCR was performed using a 7500 Real-time PCR System (Applied Biosystems) with SYBR Premix Ex Taq Kit (Takara). The following primers were used: APRIL, forward: 5'-ATTAACGCCACCTCCAAG-3', reverse: 5'-CAGCAGATAAACTCCAGCAT-3'; $\beta$-actin, forward: 5'-AGAGCCTCGCCTTTGCCGATCC-3', reverse: 5'-CTGGGCCTCGTCGCCCACATA-3'. TaqMan probes (Genepharma, ShangHai, China) were used to quantify miR-145, and miR-145 expression levels were normalized to snRNA U6. All procedures were performed in triplicate.

\section{Immunoblotting}

Cell extracts were collected in a lysis buffer (50 mM Tris-HCl [pH 7.4], $150 \mathrm{mM} \mathrm{NaCl,} 1 \%$ Triton X-100, $0.1 \%$ SDS, 1 mM EDTA and protease inhibitor cocktail). The cellular protein was size-fractionated by SDS-polyacrylamide gel electrophoresis and transferred to PVDF membranes (Bio-Rad Laboratories). After blocking with PBS containing 5\% BSA, the membrane was incubated with the appropriate primary antibody at $4{ }^{\circ} \mathrm{C}$ overnight, followed by incubation with HRP-conjugated anti-mouse or antirabbit IgG at room temperature for $2 \mathrm{~h}$. The protein bands were detected using an enhanced chemiluminescence (ECL) detection system following the manufacturer's instructions. The following primary antibodies were used: anti-APRIL, anti-MDM2 (Abcam), anti-Bcl-2, anti-Bcl-xl, anti-Bax, anti-p53, anti-pAKT, anti-AKT, anti-IкB $\alpha$, anti-p65, anti-GAPDH and anti-H3 (Cell signaling technology). All procedures were performed in triplicate.

\section{Nuclear/cytoplasmic fractionation}

Nuclear/cytoplasmic lysates were prepared using a Nuclear/Cytosol Fractionation Kit(BioVision). Cells were resuspended with $0.2 \mathrm{ml}$ CEB-A mix containing DTT and protease inhibitors, followed by incubation with $11 \mu \mathrm{l}$ CEB-B for $1 \mathrm{~min}$. Then centrifuged and collected the supernatant (cytoplasmic extract). Finally, resuspended the pellet with $100 \mu \mathrm{l}$ NEB mix, followed by centrifugation and collecting the supernatant (nuclear extract).

\section{Cell proliferation and cytotoxicity assay}

Cells (2000/well) were seeded into 96-well plates and stained at the indicated time point using the Cell Counting Kit-8 (Dojindo Laboratories), according to the instructions of the manufacturer's instructions. The optical density measured at $450 \mathrm{~nm}$ was used as an indicator of cell viability. For cytotoxicity assay, cells were treated with various concentrations of cisplatin (Selleck) dissolved in PBS. All procedures were performed in triplicate.

Colony formation

GC cells transfected with the vectors containing APRIL (or the empty vector as a control) were cultured in 6-well plates (3000 cells/well). After adherence, the cells were treated with cisplatin (27 $\mu \mathrm{M})$ for 8 hours. 
Zhi et al.: APRIL Expression in Gastric Cancer

After the treatments, the cells were washed with PBS and cultured for 3 weeks. Colonies composed of 50 or more cells were scored as survivors. Proliferating clonies were stained with crystal violet and counted. All procedures were performed in triplicate.

Construction of recombinant plasmids and lentivirus production

The full-length ORF of APRIL (753bp, NM_003808.3) was amplified from cDNA of GC cells SGC7901. The primers were as follows: forward, 5'- AGAGAATTCATGCCAGCCTCATCTCCTTT-3', reverse, 5'- AGAGGATCCTCACAGTTTCACAAACCCCA-3'. The PCR product was inserted into the expression vector pcDNA3.1/myc-His(-)B (Invitrogen). To construct the lentivirus production containing APRIL, the ORF of APRIL was subcloned into the pLenti-CMV-GFP vector (Addgene).

The synthesised DNA fragments encoding the short hairpin RNA (shRNA) used for the knockdown of endogenousAPRILwereinsertedinto thepGPU6/GFP/Neovector (GenePharma). The sequences oftheshRNAs were as follows: APRIL-shRNA, 5'- GCTGGAGTTTATCTGCTGTATCTCGAGATACAGCAGATAAACTCCAGC-3', shRNA-NC, 5'- TTCTCCGAACGTGTCACGTCTCGAGACGTGACACGTTCGGAGAA-3'. All plasmids were verified by sequencing.

\section{Luciferase reporter assay}

The 3'UTR of APRIL containing the wild or mutated miR-145 binding sequences were synthesized by Genescript (Nanjing, China). The sequences were cloned into the pGL3-control vector (Promega) to generate the APRIL 3'UTR reporter. The miRNA mimic negative control (HMC0003) and mimic of miR-145 (HMI0224) were obtained from Sigma. A reporter vector (pNF- $\kappa B-L u c)$ that contained multiple copies of the nuclear factor NF- $\kappa B$ responsive element upstream of the pTAL vector was used for monitoring NF- $\kappa B$ activity (Clontech).

Cells were seeded onto 24 -well plates and were tranfected with reporter vectors ( $0.6 \mu \mathrm{g} / \mathrm{well})$ together with phRL-TK vectors $(0.05 \mu \mathrm{g} /$ well) (Promega) using Lipofectamine 2000 reagent (Invitrogen) according to the manufacturer's protocol. The phRL-TK vectors were used for standardization of the data. Firefly and Renilla luciferase activities were measured by Dual-Luciferase reporter assay (Promega). All procedures were performed in triplicate.

Tumor xenografts and anticancer chemotherapy in vivo

Male BALB/c nude mice ( 5 weeks old) were purchased from Vitalriver (Nanjing, China). AGS-NC and AGS-APRIL stably transfected cells were inoculated subcutaneously to form the first-generation xenografts. The xenografts were serially transplanted in nude mice by inoculating tumor fragments $(2 \times 2 \times 2 \mathrm{~mm})$ subcutaneously with a trocar needle. One week after inoculation, each kind of xenografts were devided into chemotherapy group and control group. Twelve mice were used in each group according to the sample estimation with a statistical power of $90 \%$. Control group received saline only. Chemotherapy group received $7.5 \mathrm{mg} / \mathrm{kg}$ of cisplatin once per week intraperitoneally. The regimens were performed for 4 weeks [19]. Mice were sacrificed after treatment. The kinetics of tumour formation was assessed by measuring the tumour sizes every 4 days. The tumor volume was calculated using the formula: volume $=\left(\right.$ width $^{2} \times$ length $) / 2$.

\section{Heparitinase treatment}

$10 \mathrm{mU} / \mathrm{ml}$ heparitinase (Sigma) prepared in $20 \mathrm{mM}$ Tris- $\mathrm{HCl}, \mathrm{PH} 7.5$, containing $0.1 \mathrm{mg} / \mathrm{ml}$ BSA and 4 $\mathrm{mM} \mathrm{CaCl}_{2}$ was added into AGS-APRIL cells. Control incubations (AGS-NC and AGS-APRIL cells) were treated identical without the addition of enzyme. All experiments were performed in triplicate.

\section{Statistics}

The significance of APRIL expression in primary tumors compared with paired non-tumors was assessed by the paired Wilcoxon test. Probability of differences in OS was ascertained by Kaplan-Meier method, with a log-rank test probe for significance. Univariate and multivariate Cox proportional hazards regression analyses were performed to estimate the hazard ratios (HRs). The cut-off level of APRIL was evaluated by plotting $[\mathrm{t}, \mathrm{AUC}(\mathrm{t})]$ for different values of follow-up time $(\mathrm{t})$. All the statistical analyses were performed by SPSS software (version 13.0, Chicago, IL). A $p$-values $<0.05$ were considered statistically significant.

\section{KARGER}




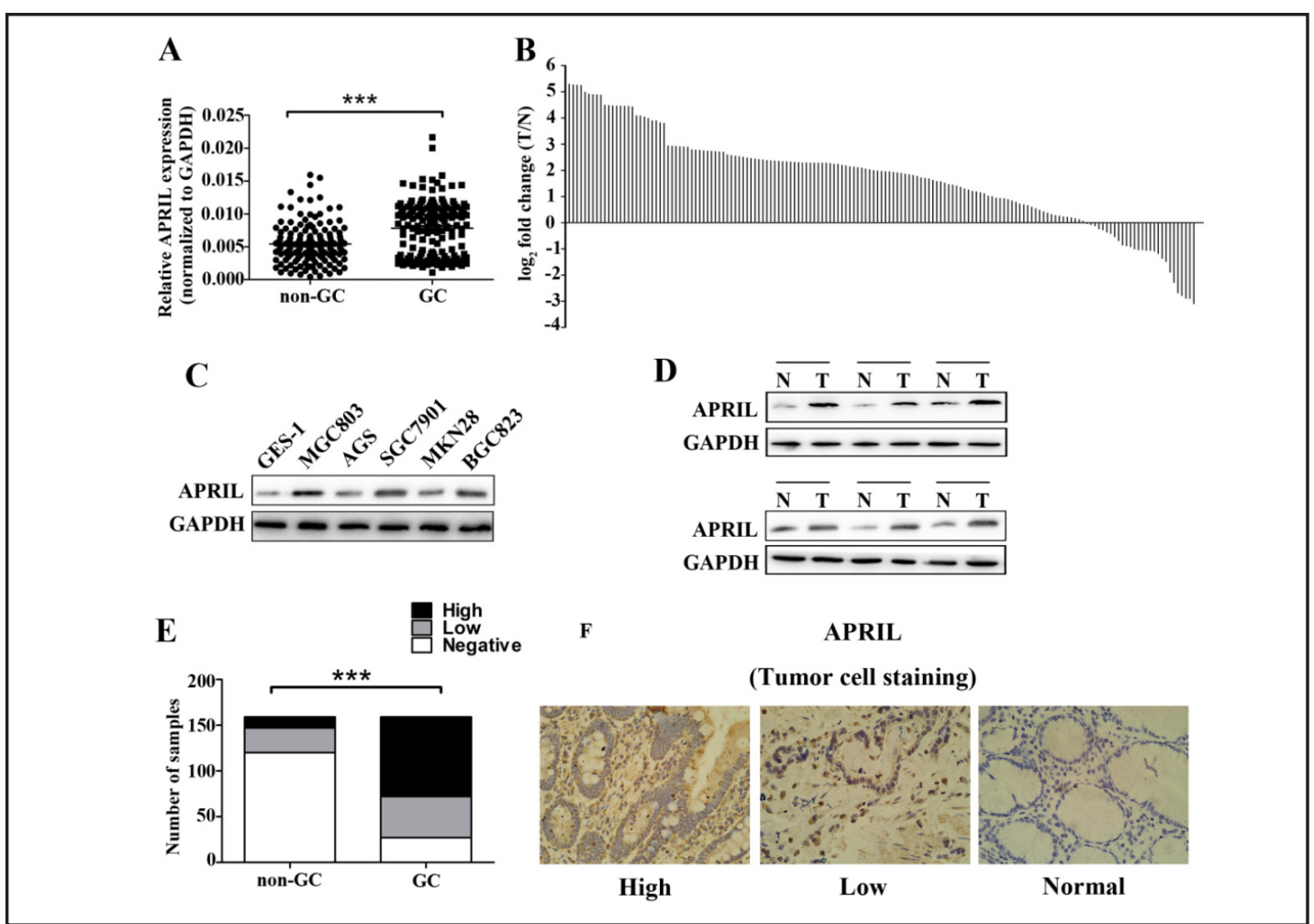

Fig. 1. APRIL mRNA and protein expression in GC and GC cell lines. (A) APRIL mRNA expression in 159 pairs of human GC and their corresponding non-tumour samples. APRIL expression levels were detected by real-time PCR and normalised to $\beta$-actin. The statistical analysis was performed using paired Wilcoxon test. (B) Paired comparison of APRIL mRNA levels between GC samples and the corresponding noncancerous samples. (C) Expression levels of APRIL determined by immunoblotting analysis in 5 GC cell lines and 1 human gastric mucosal cell line. $\beta$-actin was used as an internal control. (D) Immunoblotting analysis results of 6 pairs of GC (T) and the adjacent noncancerous tissues (N). (E) The distribution of different APRIL protein expression in tumor cells and mucosal cells detected by immunohistochemistry. (F) Representative images of positive APRIL staining (high and low) in tumor cells and negative APRIL staining in noncancerous tissues (magnification: $400 \times$ ). ${ }^{* * *} p<0.001$.

\section{Results}

APRIL is upregulated in GC and is associated with tumor size and H. pylori infection

We analysed APRIL mRNA expression levels in 159 GC patients and their paired nontumor tissues by real-time PCR. It was shown that APRIL mRNA expression was significantly upregulated in the GC tissues compared to the corresponding adjacent non-tumor samples (Fig. 1A, 1B). The APRIL expression levels of 6 pairs of typical cases, 5 human GC cell lines and 1 human gastric mucosal cell line were validated by western blotting (Fig. 1C, 1D). To further confirm APRIL expression levels in tumor cells, not in stroma, IHC was performed. It was shown that tumor cells expressed either high or low APRIL protein, while normal mucosal cells rarely expressed APRIL protein (Fig. 1F). The distribution of different APRIL expression in tumor cells and mucosal cells was shown in Fig. 1e. The protein levels of APRIL were significantly increased in tumor cells (high 87 cases, low 45 cases and negative 27 cases) and decreased in mucosal cells (high 12 cases, low 27 cases and negative 120 cases) (Fig. 1E).

In addition, we found that APRIL staining in tumor cells was significantly associated with $H$. pylori infection and tumor size (Table 2). This was confirmed by the results from real-time PCR analysis (Table 2). 
Table 2. The correlations of APRIL expression with various clinicopathological features of gastric cancer patients. IHC: Immunohistochemistry. RT-PCR: real-time polymerase chain reaction. Hp: H. pylori. ${ }^{*} p<0.05$ statistically significant difference. ${ }^{\text {athe }} 7^{\text {th }}$ AJCC staging manual

\begin{tabular}{|c|c|c|c|c|c|}
\hline \multirow{2}{*}{$\begin{array}{l}\text { Clinicopathological } \\
\text { features }\end{array}$} & \multicolumn{2}{|c|}{$\begin{array}{c}\text { APRIL expression } \\
\text { (IHC) }\end{array}$} & \multirow{2}{*}{$\begin{array}{c}p- \\
\text { value(IHC) }\end{array}$} & \multirow{2}{*}{$\begin{array}{l}\text { APRIL expression (RT-PCR, } \\
\text { mean } \pm \text { SEM) }\end{array}$} & \multirow{2}{*}{$\begin{array}{l}p \text {-value(RT- } \\
\text { PCR) }\end{array}$} \\
\hline & $\begin{array}{l}\text { Low } \\
(\mathrm{n}=72)\end{array}$ & $\begin{array}{l}\text { High } \\
(\mathrm{n}=87)\end{array}$ & & & \\
\hline \multicolumn{6}{|l|}{ Gender } \\
\hline Male & 47 & 51 & 0.417 & $0.008 \pm 0.001$ & 0.381 \\
\hline Female & 25 & 36 & & $0.007 \pm 0.001$ & \\
\hline \multicolumn{6}{|l|}{ Age(year) } \\
\hline$\geq 60$ & 43 & 40 & 0.111 & $0.007 \pm 0.001$ & 0.158 \\
\hline$<60$ & 29 & 47 & & $0.009 \pm 0.001$ & \\
\hline \multicolumn{6}{|l|}{$\mathrm{Hp}$} \\
\hline Positive & 23 & 44 & $0.024^{*}$ & $0.011 \pm 0.001$ & $0.013^{*}$ \\
\hline Negative & 49 & 43 & & $0.006 \pm 0.001$ & \\
\hline \multicolumn{6}{|l|}{ Tumor size $[\mathrm{cm}]$} \\
\hline$\geq 3$ & 31 & 53 & $0.027^{*}$ & $0.009 \pm 0.001$ & $0.044^{*}$ \\
\hline$<3$ & 41 & 34 & & $0.006 \pm 0.001$ & \\
\hline \multicolumn{6}{|l|}{ Differentiation } \\
\hline Well & 22 & 34 & 0.318 & $0.007 \pm 0.001$ & 0.321 \\
\hline Moderately and poorly & 50 & 53 & & $0.008 \pm 0.001$ & \\
\hline \multicolumn{6}{|l|}{ TNM stage $^{a}$} \\
\hline $\mathrm{I}+\mathrm{II}$ & 11 & 7 & 0.209 & $0.008 \pm 0.001$ & 0.175 \\
\hline III+IV & 61 & 80 & & $0.009 \pm 0.001$ & \\
\hline
\end{tabular}

Table 3. Multivariate Cox regression analysis of APRIL expression and other clinicopathologic variables predicting survival of gastric cancer patients. HR: hazard ratio. CI: confidence interval. ${ }^{*} p<0.05$ statistically significant difference. ${ }^{\text {at }}$ the $7^{\text {th }}$ AJCC staging manual

\begin{tabular}{lccc}
\hline Variables & HR & $95 \%$ CI & $p$-value \\
\hline Age $(\geq 60$ vs. <60) & 1.117 & $0.721-1.685$ & 0.615 \\
Gender (male vs. female) & 1.063 & $0.786-1.439$ & 0.683 \\
Tumor size ( $\geq 3$ vs. $<3 \mathrm{~cm}$ ) & 0.962 & $0.655-1.637$ & 0.785 \\
Differentiation (moderately and poorly vs. well) & 1.476 & $1.087-2.154$ & $0.019^{*}$ \\
TNM stage (III-IV vs. I-II)a & 4.215 & $2.987-6.278$ & $<0.001^{*}$ \\
APRIL expression (high vs. low) & 1.016 & $0.793-2.132$ & 0.209 \\
\hline
\end{tabular}

High expression of APRIL is correlated with poor prognosis in cisplatin-treated GC patients

We constructed Kaplan-Meier survival curves to study the relationship between APRIL protein expression and survival after surgical resection. In the analysis of overall $159 \mathrm{GC}$ cases, there was no significant relationship between APRIL protein expression in tumor cells and survival (Fig. 2A). In patients receiving 5-FU-based chemotherapy, there was no significant association either (Fig. 2B). Notably, high levels of APRIL protein in tumor cells were associated with poor survival in those patients with cisplatin-based chemotherapy (Univariate Cox regression HR =1.593, 95\% CI: 1.318-2.240; log-rank $P=0.037$ ) (Fig. 2C). These results indicated that APRIL might induce cisplatin resistance in GC tumor cells.

Next, we investigated the influence of APRIL expression and other clinicopathological parameters on overall survival using multivariate Cox regression analysis. Our results showed that differentiation and TNM stage were independent factors for overall survival in GC patients (Table 3). 
Fig. 2. Prognostic value of APRIL in GC patients. (A) In all 159 GC patients. (B) In 148 GC patients receiving 5-FU-based treatment. (C) In 129 GC patients receiving cisplatin-based treatment. Hazard ratio (HR) was calculated by Univariate Cox regression analysis. (D) The values of area under curve (AUC) at different IRS of APRIL for overall survival.
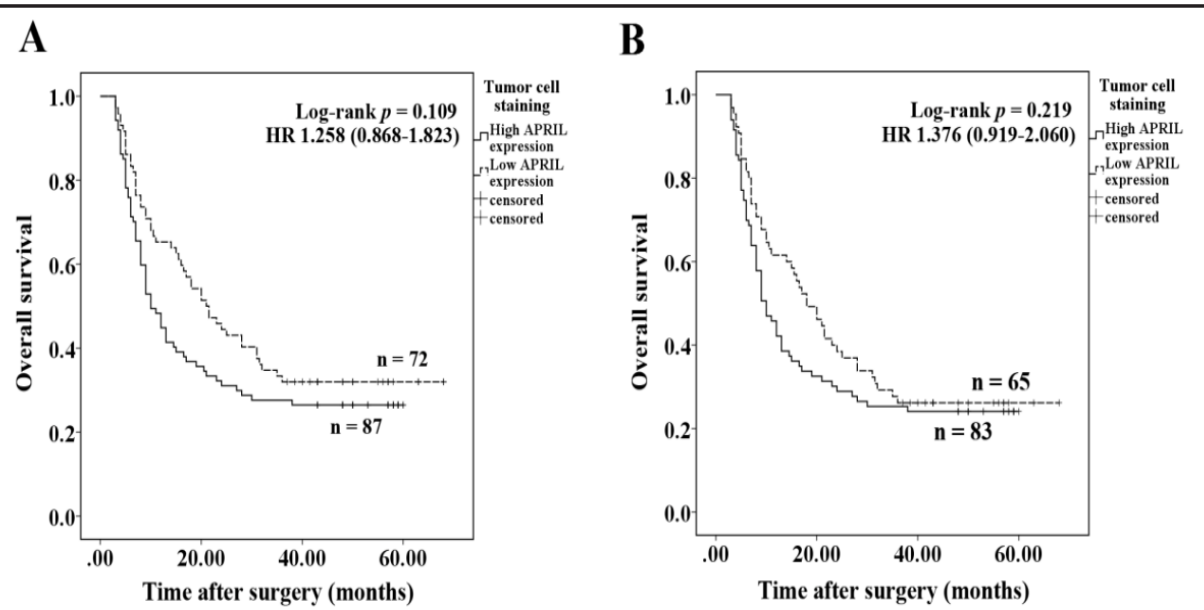

C

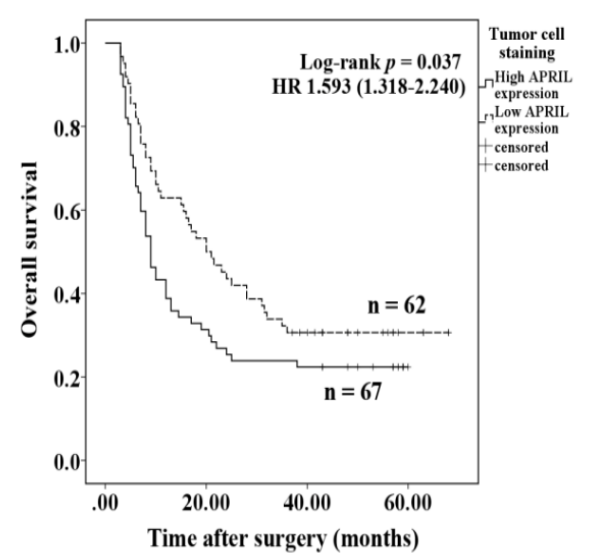

D

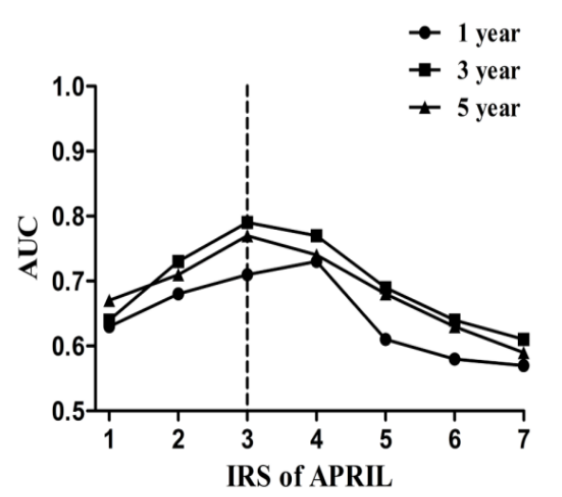

APRIL promotes resistance to cisplatin in human GC cells

We chose high APRIL-expression GC cell line SGC7901 and low APRIL-expression GC cell line AGS to generate stable APRIL-knockdown cell line (SGC7901-shRNA) and stable APRILoverexpression cell line (AGS-APRIL). AGS-APRIL cells displayed a 3-fold increase of APRIL protein compared with AGS-NC cells. SGC7901-shRNA cells showed a 91\% downregulation of APRIL protein compared with SGC7901-NC cells (Fig. 4A).

Measurement of $\mathrm{IC}_{50}$ was performed to evaluate the sensitivity of AGS-APRIL cells or SGC7901-shRNA cells to cisplatin. It was showed that the silence of APRIL in SGC7901 leaded to an increased sensitivity to cisplatin (IC F0 $_{50}$ SGC7901-shRNA, $2.64(2.38-3.05) \mu \mathrm{M}$ vs. SGC7901-NC, 4.83 (4.46-5.23) $\mu \mathrm{M}$ ). In contrast, overexpression of APRIL in AGS showed an decreased sensitivity to ciaplatin (IC I0 : AGS-APRIL, 37.20 (33.73-41.02) $\mu \mathrm{M}$ vs. AGS-NC, 27.32 (24.86-30.03) $\mu \mathrm{M}$ ) (Fig. 3A).

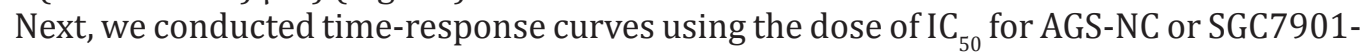
NC. The response curve of AGS-APRIL showed more resistant to cispaltin than AGS-NC from $24 \mathrm{~h}$ to $72 \mathrm{~h}$, whereas SGC7901-shRNA had a better response curve compared to SGC7901-NC from $12 \mathrm{~h}$ to $72 \mathrm{~h}$ (Fig. $3 \mathrm{~B}$ ).

To further determine the long-term effects of APRIL on cisplatin-inhibited cell proliferation, colony formation assay was performed. As shown in Fig. 3C, although cisplatin induced a dramatic inhibition of colony formation in AGS-NC cells, only a partial inhibition was observed in AGS-APRIL cells when cells were treated with the same concentration of cisplatin (survival rate: mean number of colonies in cisplatin-treated group/ mean number of colonies in saline-treated group). 


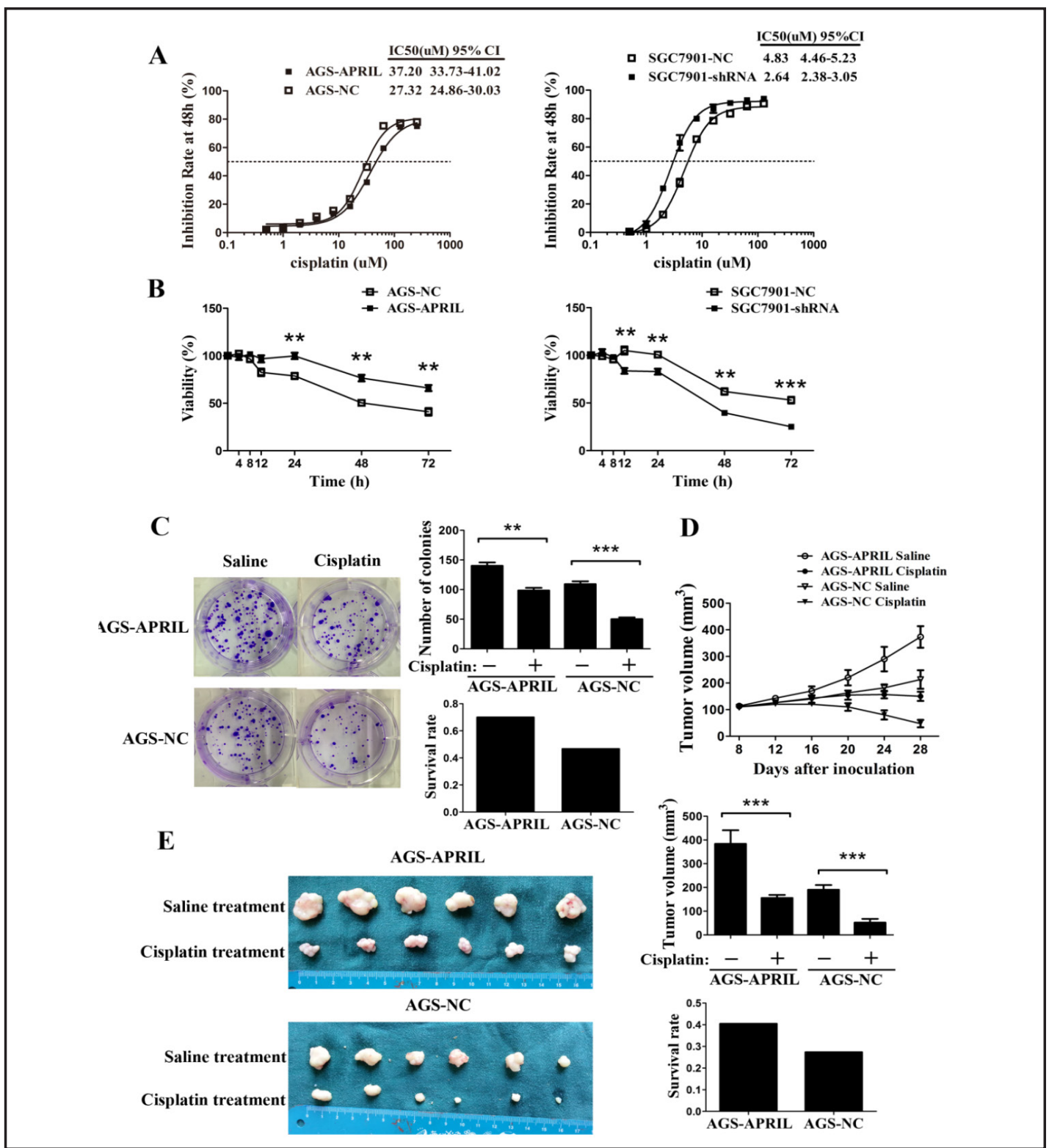

Fig. 3. The influence of APRIL on chemo-resistance in GC cells. (A) The half inhibitory concentration (IC50) of cisplatin was determined by cell proliferation assay. Cells were treated with increasing concentrations of cisplatin for 48h. (B) Time-response curves were detected using the dose of IC50 for AGS-NC and SGC7901NC, respectively. (C) The long-term effect of APRIL on chemo-resistance was studied by colony formation (survival rate: mean number of colonies in cisplatin-treated group/ mean number of colonies in saline-treated group). (D) The growth curves of xenografts in nude mice during chemotherapy. The tumor volume was calculated using the formula: volume $=($ width $2 \times$ length $) / 2$. $(E)$ The final xenografts after chemotherapy in nude mice (survival rate: mean tumor volume in cisplatin-treated xenografts/mean tumor volume in saline-treated xenografts). ${ }^{* *} p<0.01,{ }^{* * *} p<0.001$.

\section{APRIL attenuates therapeutic efficacy in nude mice treated with cisplatin}

In both two treatment groups with cisplatin, tumor growths were significantly inhibited, as shown in Fig. 3D and 3E. The growth rate of cisplatin-treated AGS-NC cells began to go down on day 16, while that of cisplatin-treated AGS-APRIL cells kept rising in the first 24 days and went down after 24 days (Fig. 3D). After treatment, the survival rate of AGS-APRIL xenografts (mean tumor volume in cisplatin-treated AGS-APRIL xenografts/mean tumor 
Fig. 4. Activation of $N F-\kappa B$ pathway in AGS-APRIL and SGC7901-shRNA cells. (A) Expression levels of pAKT, AKT, I $\mathrm{K} \mathrm{B} \alpha$ and p65 were analyzed by western blotting. (B, C) p65 extracted from cytoplasma and nuclear was detected by western blotting. (D) Luciferase activity of pNF- $\kappa \mathrm{B}-\mathrm{Luc}$ that contained multiple copies of the nuclear factor NF- $\kappa B$ responsive element was used for monitoring NF- $\kappa B$ activity. The phRL-TK vectors were used for standardization of the data. (E) Expression of Bcl2, Bcl-xl, Bax, p53 and MDM2 were analyzed by western blotting. (F) The density of each band was measured and ratios

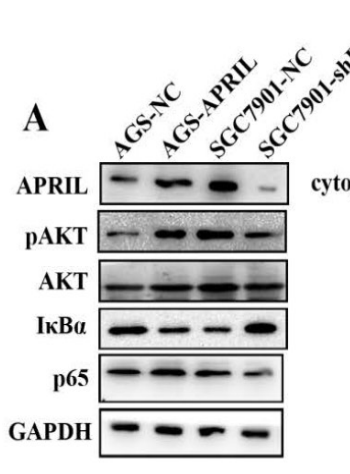

D

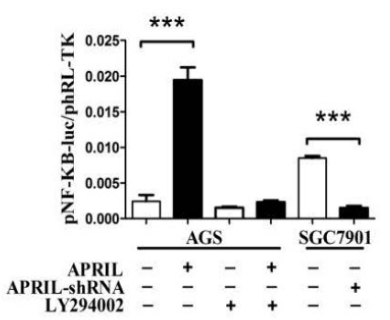

F
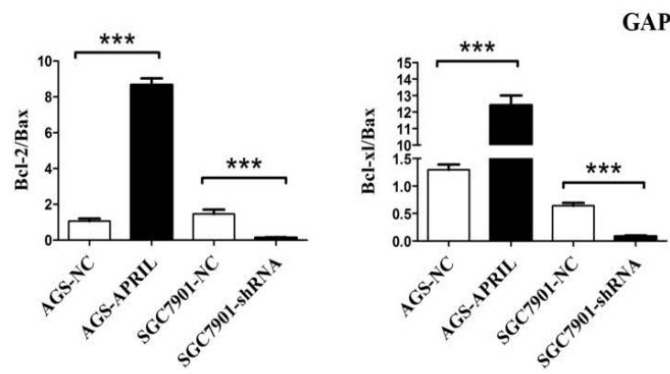

G APRIL

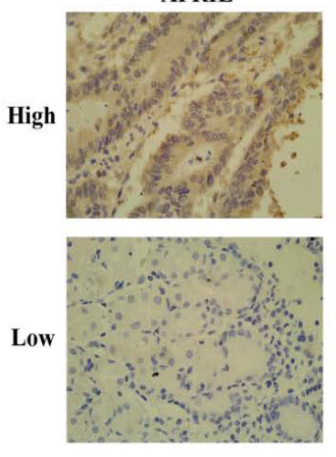

B

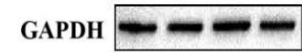

nuclear p 65

H3

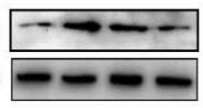

E cytoplasmic p65 $-3-\infty-$ GAPDH $-\infty$ nuclear p $65-\infty--$

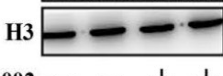
LY294002 - - + +

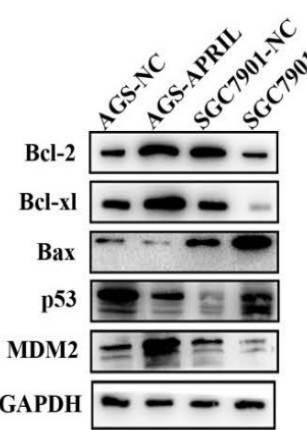

of Bcl-2/Bax and Bcl-xl/Bax were calculated. (G) Representative images of APRIL, Bcl-2 and Bcl-xl in GC (magnification: $400 \times$ ). ${ }^{* *} p<0.01,{ }^{* * *} p<0.001$.

volume in saline-treated AGS-APRIL xenografts) was higher than that of AGS-NC xenografts (Fig. 3E). These results indicated that APRIL exhibited a significant effect on inhibiting the therapeutic efficacy of cisplatin in vivo.

\section{APRIL induces cisplatin resistance via activating NF- $\kappa B$ pathway}

As increasing amounts of data demonstrate that the activation of NF- $\kappa B$ pathway plays an indispensible role in APRIL-mediated chronic lymphocytic leukemia B-cell survival $[20,21]$, we investigated the relationship between APRIL and NF- $\kappa B$ pathway involved in 
A

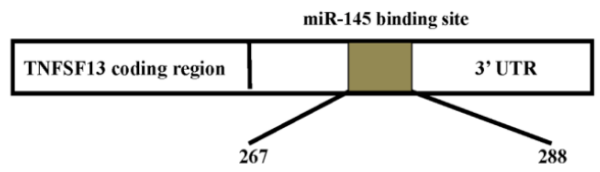

wt TNFSF13 3'UTR: 5' ACCUGGUCGGGGCCCACUGGAA3,

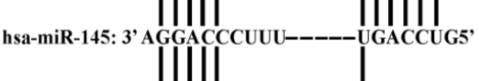

mut TNFSF13 3'UTR: 5' ACCUGGUCGGGGCCCAGCCAGA 3,
B

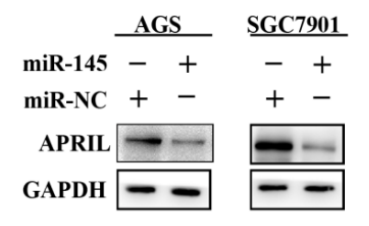

C

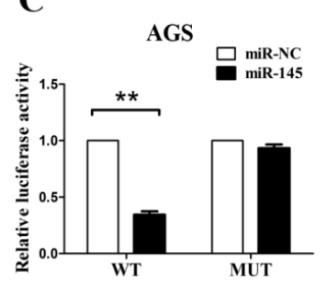

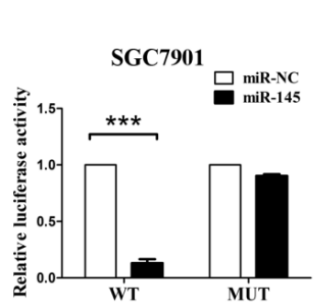

D

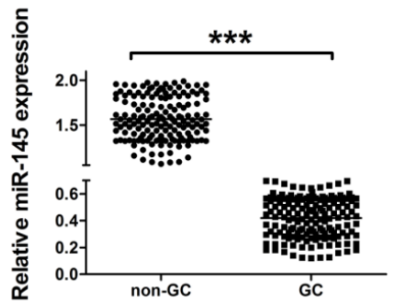

F

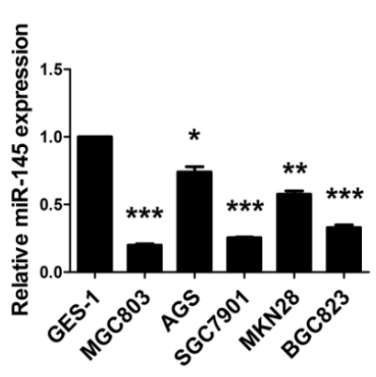

Fig. 5. APRIL was regulated by miR-145. (A) The APRIL 3'UTR regions containing the wild-type or mutant binding site for miR-145 are shown. (B) Western blotting was used to analyze the expression levels of APRIL after transfecting miR-NC or miR-145 mimics. (C) Relative APRIL luciferase activity was analyzed after the wild-type or mutant 3'UTR reporter plasmids co-transfected with miR-NC or miR-145 mimics. (D) Real-time PCR was used to detect the expression levels of miR-145 in GC and non-tumour specimens. The statistical analysis was performed using paired Wilcoxon test. (E) The scatter plots show the expression levels of miR-145 and APRIL in GC samples. Linear regression analysis was used to measure the association between miR-145 and APRIL. (F) Real-time PCR analysis of miR-145 in gastric mucosal epithelial cell line (GES-1) and gastric cancer cell lines. ${ }^{* *} p<0.01,{ }^{* * *} p<0.001$.

GC cells. APRIL was recently reported to activate pAKT to promote colorectal cancer cells proliferation [13], and the phosphorylated AKT could subsequently upregulate the activity of NF- $\mathrm{B}$ [22]. Our results showed that APRIL overexpression in AGS cells significantly increased the phosphorylation of AKT, while silence of APRIL in SGC7901 cells significantly decreased pAKT (Fig. 4A). Activation of the canonical NF- $\kappa B$ pathway depends on IKKdependent degradation of IкB $\alpha$ [23], we found that APRIL overexpression promoted the degradation of $I \kappa B \alpha$ and consequently upregulated total p65. In contrary, knockdown of APRIL significantly increased I $\mathrm{B} \alpha$ and decreased total p65 (Fig. 4A). In addition, Nuclear/ cytoplasmic fractionation assay indicated that APRIL overexpression induced nuclear translocation of p65, whereas APRIL silence attenuated its nuclear translocation (Fig. 4B). LY294002 inhibited the activation of AKT and consequently attenuated nuclear translocation of p65 caused by APRIL overexpression (Fig. 4C). Luciferase reporter assay also confirmed

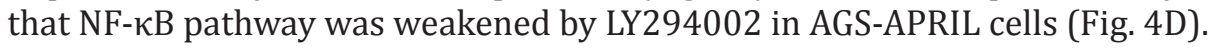


A

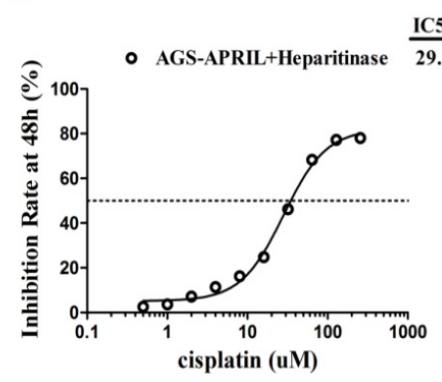

B

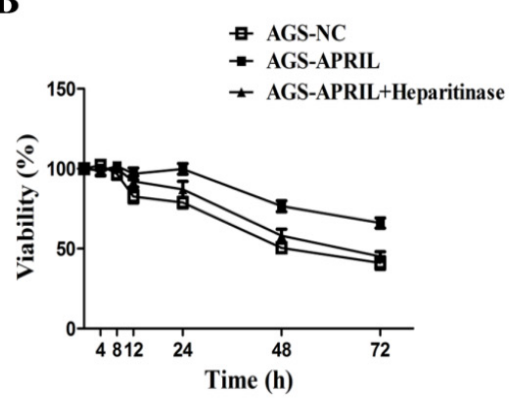

C

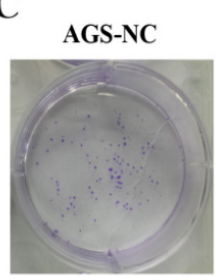

D

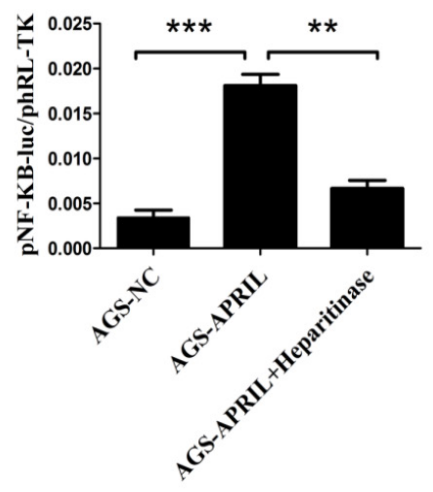

$\frac{\text { IC50(uM) } 95 \% \text { CI }}{29.2126 .75-31.99}$ 
miR-145 regulates APRIL expression through directly targeting its 3'UTR

To understand the regulation of APRIL in GC cells, we used three algorithms (TargetScan, PicTar and miRanda) to predict miRNAs which might target APRIL. Two putative miRNAs (miR-145 and miR-199ab-5p) were figured out. Given that miR-145 has been reported to be downregulated and implicated in suppressing invasion, metastasis and angiogenesis in gastric cancer [25-27], we hypothesized that miR-145 downregulated APRIL expression in GC cells. The luciferase reporter assay was employed to validate the hypothesis. Wildtype and mutant APRIL 3'UTR containing putative target sites of miR-145 were cloned into reporter plasmids respectively (Fig. 5A). The results revealed that miR-145 significantly declined the activity of the luciferase reporter gene fused to the APRIL 3'UTR. Mutation of the putative miR-145 binding sites in the 3'UTR of APRIL abrogated luciferase responsiveness to miR-145 (Fig. 5C). Western blot showed that APRIL protein levels were inhibited in miR-145 mimics-transfected cells (Fig. 5B). Furthermore, we confirmed that miR-145 was significantly down-regulated in GC and GC cell lines (Fig. 5D, 5F) and the level of miR-145 was inversely correlated with APRIL mRNA (Fig. 5E).

HSPG are required for APRIL-induced cisplatin resistance and NF- $\kappa B$ translocation

To demonstrate the contribution of heparan sulfate proteoglycans (HSPG) in APRILinduced cisplatin resistance, we evaluated $\mathrm{IC}_{50}$, time-response curves, colony formation, luciferase activity of pNF- $\kappa B$-Luc and NF- $\kappa B$ translocation in AGS-APRIL cells treated with heparitinase (Fig. 6). As shown in Fig. 6A, APRIL-induced resistance to cisplatin was markedly inhibited by heparitinase (IC50: AGS-APRIL+Heparitinase, 28.21 (25.68-30.99) $\mu \mathrm{M}$ vs. AGS-APRIL, 37.20 (33.73-41.02) $\mu \mathrm{M}$ ). In Fig 6B, we used two-way ANOVA to analyze the data. When compared to AGS-APRIL cells, AGS-APRIL+ Heparitinase cells showed more sensitive to cisplatin from $24 \mathrm{~h}$ to $72 \mathrm{~h}(t=2.867$ and $P<0.05$ in $24 \mathrm{~h}, t=4.135$ and $P<$ 0.001 in $48 \mathrm{~h}, t=4.685$ and $P<0.001$ in $72 \mathrm{~h}$ ). However, when compared to AGS-NC cells, AGS-APRIL+ Heparitinase cells did not get significant differences. Colony formation assay was also performed to determine the long-term effects of heparitinase. As shown in Fig. 6C, heparitinase attenuated APRIL-induced colony formation in AGS-APRIL cells exposure to cisplatin. Luciferase reporter assay confirmed that NF- $\kappa B$ pathway was weakened by heparitinase treatment in AGS-APRIL cells (Fig. 6D). In addition, the nuclear translocation of NF-KB induced by APRIL was diminished by heparitinase treatment (Fig. 6E). These results indicated that the effects of APRIL were mediated by HSPG and APRIL-HSPG binding is necessary for NF-KB translocation.

\section{Discussion}

The survival of resectable GC has shown some progress by more precise surgery and combining chemotherapy with targeted treatment [28, 29]. However, the improvements are small and confined to ill-defined subgroups, except for HER-2 positive GC. Thus, identification of novel prognostic biomarkers as well as therapeutic targets is urgent for GC patients. In the present study, we identified a novel chemo-resistance biomarker APRIL for gastric cancer, which is a tumor-necrosis factor (TNF) family member and is a novel cytokine crucial in sustaining lymphocytic leukemia B cell survival and proliferation $[3,6]$.

The tumor-necrosis factor (TNF) family of 19 ligands and 29 receptors orchestrates a wide range of biological functions [30]. Two new TNF ligands were found via expressed sequence tag (EST) database: APRIL and BAFF $[3,31]$. The discovery of the new network between these two TNF ligands and their receptors (TACI, BCMA and BAFF-R) triggered an avalanche of research. Although much effort has focused on BAFF, the functions of APRIL have received less attention. High levels of APRIL mRNA were expressed in cancers of gastrointestinal tract suggesting a close interrelationship between APRIL and gastrointestinal cancers [3]. Recently, some malignant functions of APRIL have been discovered in colorectal cancer [12, 13, 32]. Our previous study explored that knockdown of APRIL promoted apoptosis of gastric cancer 
cells and down-regulated the receptor tyrosine kinases [15]. In this study, we investigated the roles of APRIL expression in GC progression and prognosis. APRIL mRNA extracted from GC tissues was significantly upregulated compared to the corresponding adjacent non-tumor samples. Given that APRIL is not only expressed in carcinoma cells, but also in immune cells infiltrated in cancer [4], we further detected APRIL protein expression in tumor cells by IHC. It was shown that tumor cells expressed either high or low APRIL protein, while normal mucosal cells rarely expressed APRIL protein. In addition, we found that APRIL staining in tumor cells was significantly associated with $H$. pylori infection and tumor size, which was confirmed by the results from real-time PCR analysis. In the analysis of overall 159 GC cases, there was no significant relationship between APRIL protein expression in tumor cells and OS. In patients receiving 5-FU-based chemotherapy, there was no significant association either. When it comes to those patients with cisplatin-based chemotherapy, high levels of APRIL protein in tumor cells were associated with poor OS. These results supported further investigation of a putative functional role for APRIL in clinical cisplatin chemo-resistance. Both in vitro and in vivo assays confirmed that APRIL induced cisplatin resistance in GC tumor cells.

APRIL is shown to bind to TACI and BCMA, but not BAFF-R which only binds to BAFF, and to activate the nuclear factor- $\mathrm{\kappa B}(\mathrm{NF}-\kappa \mathrm{B})$ pathway $[33,34]$. All known receptors have been shown to be expressed on B cells as well as carcinoma cells [35]. On the other hand, APRIL interacts with heparan sulfate proteoglycans (HSPG), which are structurally unrelated to TNF receptors and are likely a third receptor for APRIL [36-38]. In the present study, we showed that APRIL activated the canonical NF- $\kappa B$ pathway through phosphorylation of AKT. Thus, the NF- $\kappa B$ pathway target genes, such as Bcl-2 and Bcl-xl [21], were upregulated and contributed to cisplatin resistance. In addition, we also explored the mechanism of regulating APRIL expression in GC cells. We predicted two candidates of putative miRNAs (miR-145 and miR-199ab-5p) using three algorithms (TargetScan, PicTar and miRanda). Given that miR-145 has been reported to be downregulated and implicated in suppressing invasion, metastasis and angiogenesis in gastric cancer [25-27], we hypothesized that miR145 downregulated APRIL expression in GC cells. The luciferase reporter assay validated this hypothesis. Western blot also showed that APRIL protein levels were inhibited in miR145 mimics-transfected cells. Our data suggested that HSPG function as the main receptor for APRIL in GC cells. Also, our studies showed that the APRIL-HSPG interaction triggers APRIL-mediated NF-KB activation on GC cells.

In conclusion, our results demonstrated that high levels of APRIL expression significantly correlated with unfavorable prognosis in the patients receiving cisplatin-based chemotherapy. The canonical NF- $\kappa$ B pathway was involved in APRIL-induced cisplatin resistance in GC cells. Thus, APRIL may serve as a promising prognostic marker and a therapeutic target for GC. So far, some novel therapeutic agents designed to neutralize APRIL has been tested in clinical trials or in preclinical experimentation $[39,40]$. Our study encourages further clinical trials to prove that neutralizing APRIL will be an effective therapy for GC patients.

\section{Acknowledgments}

This work was supported by the Nanjing Medical Technology Development Project (YKK12075) and Jiangsu Provincial Graduates Innovative Project (CXZZ12_0584).

\section{Disclosure Statement}

The authors declare that they have no conflict of interest.

\section{References}

1 Ferlay J, Shin HR, Bray F, Forman D, Mathers C, Parkin DM: Estimates of worldwide burden of cancer in 2008: GLOBOCAN 2008. Int J Cancer 2010;127:2893-2917. 
Zhi et al.: APRIL Expression in Gastric Cancer

2 Cunningham D, Allum WH, Stenning SP, Thompson JN, Van de Velde CJ, Nicolson M, Scarffe JH, Lofts FJ, Falk SJ, Iveson TJ, Smith DB, Langley RE, Verma M, Weeden S, Chua YJ, MAGIC Trial Participants: Perioperative chemotherapy versus surgery alone for resectable gastroesophageal cancer. N Engl J Med 2006;355:11-20.

-3 Hahne M, Kataoka T, Schröter M, Hofmann K, Irmler M, Bodmer JL, Schneider P, Bornand T, Holler N, French LE, Sordat B, Rimoldi D, Tschopp J: APRIL, a new ligand of the tumor necrosis factor family, stimulates tumor cell growth. J Exp Med 1998;188:1185-1190.

4 Ng LG, Mackay CR, Mackay F: The BAFF/APRIL system: life beyond B lymphocytes. Mol Immunol 2005;42:763-772.

-5 Dillon SR, Gross JA, Ansell SM, Novak AJ: An APRIL to remember: novel TNF ligands as therapeutic targets. Nat Rev Drug Discov 2006;5:235-246.

6 von Bulow GU, Bram RJ: NF-AT activation induced by a CAML-interacting member of the tumor necrosis factor receptor superfamily. Science 1997;278:138-141.

-7 Laâbi Y, Gras MP, Carbonnel F, Brouet JC, Berger R, Larsen CJ, Tsapis A: A new gene, BCM, on chromosome 16 is fused to the interleukin 2 gene by a $\mathrm{t}(4 ; 16)(\mathrm{q} 26 ; \mathrm{p} 13)$ translocation in a malignant $\mathrm{T}$ cell lymphoma. EMBO J 1992;11:3897-3904.

-8 Schwaller J, Schneider P, Mhawech-Fauceglia P, McKee T, Myit S, Matthes T, Tschopp J, Donze O, Le Gal FA, Huard B: Neutrophil-derived APRIL concentrated in tumor lesions by proteoglycans correlates with human B-cell lymphoma aggressiveness. Blood 2007;109:331-338.

-9 Kern C, Cornuel JF, Billard C, Tang R, Rouillard D, Stenou V, Defrance T, Ajchenbaum-Cymbalista F, Simonin PY, Feldblum S, Kolb JP: Involvement of BAFF and APRIL in the resistance to apoptosis of B-CLL through an autocrine pathway. Blood 2004;103:679-688.

10 Moreaux J, Legouffe E, Jourdan E, Quittet P, Rème T, Lugagne C, Moine P, Rossi JF, Klein B, Tarte K: BAFF and APRIL protect myeloma cells from apoptosis induced by interleukin 6 deprivation and dexamethasone. Blood 2004;103:3148-3157.

11 Mhawech-Fauceglia P, Allal A, Odunsi K, Andrews C, Herrmann FR, Huard B: Role of the tumour necrosis family ligand APRIL in solid tumour development: Retrospective studies in bladder, ovarian and head and neck carcinomas. Eur J Cancer 2008;44:2097-2100.

-12 Petty RD, Samuel LM, Murray GI, MacDonald G, O'Kelly T, Loudon M, Binnie N, Aly E, McKinlay A, Wang W, Gilbert F, Semple S, Collie-Duguid ES: APRIL is a novel clinical chemo-resistance biomarker in colorectal adenocarcinoma identified by gene expression profiling. BMC Cancer DOI: 10.1186/1471-2407-9-434.

13 Wang G, Wang F, Ding W, Wang J, Jing R, Li H, Wang X, Wang Y, Ju S, Wang H: APRIL induces tumorigenesis and metastasis of colorectal cancer cells via activation of the PI3K/Akt pathway. PLoS One 2013;8:e55298.

14 Okano H, Shiraki K, Yamanaka Y, Inoue H, Kawakita T, Saitou Y, Yamaguchi Y, Enokimura N, Ito K, Yamamoto N, Sugimoto K, Murata K, Nakano T: Functional expression of a proliferation-related ligand in hepatocellular carcinoma and its implications for neovascularization. World J Gastroenterol 2005;11:46504654.

15 Ni SZ, Cao HY, Chen Z, Zhu Y, Xu ZK: siRNA interference with a proliferation-inducing ligand gene in the Sgr7901 gastric carcinoma cell line. Asian Pac J Cancer Prev 2012;13:1511-1514.

16 Jiang B, Li Z, Zhang W, Wang H, Zhi X, Feng J, Chen Z, Zhu Y, Yang L, Xu H, Xu Z: miR-874 Inhibits cell proliferation, migration and invasion through targeting aquaporin-3 in gastric cancer. J Gastroenterol DOI: 10.1007/s00535-013-0851-9.

17 Wang S, Wu X, Chen Y, Zhang J, Ding J, Zhou Y, He S, Tan Y, Qiang F, Bai J, Zeng J, Gong Z, Li A, Li G, Røe OD, Zhou J: Prognostic and predictive role of JWA and XRCC1 expressions in gastric cancer. Clin Cancer Res 2012;18:2987-2996.

18 Mackintosh KA, Fairclough SJ, Stratton G, Ridgers ND: A calibration protocol for population-specific accelerometer cut-points in children. PLoS One 2012;7:e36919.

-19 Sakurai Y, Uraguchi T, Imazu H, Hasegawa S, Matsubara T, Ochiai M, Funabiki T: Changes in thymidylate synthase and its inhibition rate and changes in dihydropyrimidine dehydrogenase after the administration of 5-fluorouracil with cisplatin to nude mice with gastric cancer xenograft SC-1-NU. Gastric Cancer 2004; 7:110-116.

-20 Endo T, Nishio M, Enzler T, Cottam HB, Fukuda T, James DF, Karin M, Kipps TJ: BAFF and APRIL support chronic lymphocytic leukemia B-cell survival through activation of the canonical NF-kappaB pathway. Blood 2007;109:703-710. 
Zhi et al.: APRIL Expression in Gastric Cancer

21 Hoesel B, Schmid JA: The complexity of NF-kappaB signaling in inflammation and cancer. Mol Cancer 2013;12:86.

22 Huang GL, Luo Q, Rui G, Zhang W, Zhang QY, Chen QX, Shen DY: Oncogenic activity of retinoic acid receptor gamma is exhibited through activation of the Akt/NF-kappaB and Wnt/beta-catenin pathways in cholangiocarcinoma. Mol Cell Biol 2013;33:3416-3425.

23 Karin M: Nuclear factor-kappaB in cancer development and progression. Nature 2006;441:431-436.

24 Muller PA, Vousden KH: p53 mutations in cancer. Nat Cell Biol 2013;15:2-8.

25 Takagi T, Iio A, Nakagawa Y, Naoe T, Tanigawa N, Akao Y: Decreased expression of microRNA-143 and -145 in human gastric cancers. Oncology 2009;77:12-21.

26 Gao P, Xing AY, Zhou GY, Zhang TG, Zhang JP, Gao C, Li H, Shi DB: The molecular mechanism of microRNA-145 to suppress invasion-metastasis cascade in gastric cancer. Oncogene 2013;32:491-501.

27 Zheng L, Pu J, Qi T, Qi M, Li D, Xiang X, Huang K, Tong Q: miRNA-145 targets v-ets erythroblastosis virus E26 oncogene homolog 1 to suppress the invasion, metastasis, and angiogenesis of gastric cancer cells. Mol Cancer Res 2013;11:182-1893.

28 Bang YJ, Van Cutsem E, Feyereislova A, Chung HC, Shen L, Sawaki A, Lordick F, Ohtsu A, Omuro Y, Satoh T, Aprile G, Kulikov E, Hill J, Lehle M, Rüschoff J, Kang YK; ToGA Trial Investigators: Trastuzumab in combination with chemotherapy versus chemotherapy alone for treatment of HER2-positive advanced gastric or gastro-oesophageal junction cancer (ToGA): a phase 3, open-label, randomised controlled trial. Lancet 2010;376:687-697.

29 Marrelli D, Roviello F, De Stefano A, Fotia G, Giliberto C, Garosi L, Pinto E: Risk factors for liver metastases after curative surgical procedures for gastric cancer: a prospective study of 208 patients treated with surgical resection. J Am Coll Surg 2004;198:51-58.

-30 Bodmer JL, Schneider P, Tschopp J: The molecular architecture of the TNF superfamily. Trends Biochem Sci 2002;27:19-26.

- 31 Schneider P, MacKay F, Steiner V, Hofmann K, Bodmer JL, Holler N, Ambrose C, Lawton P, Bixler S, AchaOrbea H, Valmori D, Romero P, Werner-Favre C, Zubler RH, Browning JL, Tschopp J: BAFF, a novel ligand of the tumor necrosis factor family, stimulates B cell growth. J Exp Med 1999;189:1747-1756.

32 Wang J, Ding W, Sun B, Jing R, Huang H, Shi G, Wang H: Targeting of colorectal cancer growth, metastasis, and anti-apoptosis in BALB/c nude mice via APRIL siRNA. Mol Cell Biochem 2012;363:1-10.

-33 Yan M, Marsters SA, Grewal IS, Wang H, Ashkenazi A, Dixit VM: Identification of a receptor for BLyS demonstrates a crucial role in humoral immunity. Nat Immunol. 2000;1:37-41.

-34 Mackay F, Ambrose C: The TNF family members BAFF and APRIL: the growing complexity. Cytokine Growth Factor Rev 2003;14:311-324.

-35 Pelekanou V, Notas G, Theodoropoulou K, Kampa M, Takos D, Alexaki VI, Radojicic J, Sofras F, Tsapis A, Stathopoulos EN, Castanas E: Detection of the TNFSF members BAFF, APRIL, TWEAK and their receptors in normal kidney and renal cell carcinomas. Anal Cell Pathol (Amst) 2011;34:49-60.

-36 Ingold K, Zumsteg A, Tardivel A, Huard B, Steiner QG, Cachero TG, Qiang F, Gorelik L, Kalled SL, AchaOrbea H, Rennert PD, Tschopp J, Schneider P: Identification of proteoglycans as the APRIL-specific binding partners. J Exp Med 2005;201:1375-1383.

- 37 Hendriks J, Planelles L, de Jong-Odding J, Hardenberg G, Pals ST, Hahne M, Spaargaren M, Medema JP: Heparan sulfate proteoglycan binding promotes APRIL-induced tumor cell proliferation. Cell Death Differ 2005;12:637-648.

-38 Sakurai D, Hase H, Kanno Y, Kojima H, Okumura K, Kobata T: TACI regulates IgA production by APRIL in collaboration with HSPG. Blood 2007;109:2961-2967.

-39 Gross JA, Dillon SR, Mudri S, Johnston J, Littau A, Roque R, Rixon M, Schou O, Foley KP, Haugen H, McMillen S, Waggie K, Schreckhise RW, Shoemaker K, Vu T, Moore M, Grossman A, Clegg CH: TACI-Ig neutralizes molecules critical for B cell development and autoimmune disease impaired B cell maturation in mice lacking BLyS. Immunity 2001;15:289-302.

40 Patel DR, Wallweber HJ, Yin J, Shriver SK, Marsters SA, Gordon NC, Starovasnik MA, Kelley RF: Engineering an APRIL-specific B cell maturation antigen. J Biol Chem 2004;279:16727-16735. 